\title{
Switched Parasitic Dielectric Resonator Antenna Array Using Capacitor Loading for 5G Applications.
}

\author{
N. H. Shahadan ${ }^{1,2}$, M. R. Kamarudin ${ }^{2}$, M. H. Jamaluddin ${ }^{2}$, M. Khalily ${ }^{3}$ and M. Jusoh ${ }^{4}$ \\ ${ }^{1}$ Department of Polytechnic, Ministry of Education Malaysia, Presint 4, 62100 W.P. Putrajaya, Malaysia. \\ Email: ayumaui2@gmail.com \\ ${ }^{2}$ Wireless Communication Centre (WCC), Universiti Teknologi Malaysia, UTM Johor, Johor 81310, Malaysia. \\ Email: ramlee@fke.utm.my \\ ${ }^{3}$ Institute for Communication Systems (ICS), Home of the 5G Innovation Centre, Department of Electronic Engineering, \\ University of Surrey, Guildford GU2 7XH, United Kingdom. \\ Email: m.khalily@s,surrey.ac.uk \\ ${ }^{4}$ School of Computer and Communication Engineering, Universiti Malaysia Perlis. \\ Email: muzammil@unimap.edu.my
}

\begin{abstract}
This paper presents a brief account of the findings on a switched parasitic dielectric resonator antenna (DRA) array excited in a higher-order mode. The scanning phase can be changed by using switching technique and capacitor loading at the parasitic element. The driven DR and parasitic DRs have a dielectric constant of 10 and were fed by a microstrip slot aperture. The impact of mutual coupling on the reflection coefficient was examined through a numerical calculation which combines both ANSYS HFSS and MATLAB. This phased array was shown to be able to steer the antenna beam from $\mathbf{- 2 6}$ degrees to +26 degrees at $15 \mathrm{GHz}$, which is considered suitable for $\mathbf{5 G}$ applications. The impedance matching was maintained at all beam steering angles and a bandwidth of $2.6 \mathrm{GHz}$ has been achieved.
\end{abstract}

Index Terms-Dielectric resonator antenna, higher-order mode, phased array, parasitic element, 5G.

\section{INTRODUCTION}

Telecommunication technology has evolved rapidly from the first generation $(1 \mathrm{G})$ to the fifth generation $(5 \mathrm{G})$ due to the increasing demand for unlimited access to information and sharing of data. 5G technology [1] needs a high gain and steerable beam directional antenna and wide bandwidth to meet all the requirements of a long distance communication [2]. In [3], the spectrum bands up to $6 \mathrm{GHz}$ are considered ideal for $5 \mathrm{G}$ applications. Due to that, this work has proposed $15 \mathrm{GHz}$ as the antenna operating frequency.

Phased array design allows for more directional antennas which are electrically steerable. However, the phase shifters are costly and require complicated feeding networks which will result in large losses at higher frequencies [4]. Therefore, there is a need to develop low cost phased arrays using different approaches. Some efforts have been taken [5]-[7] to develop steerable antenna without the need of phase shifters, namely electronically steerable passive array radiator (ESPAR). The ESPAR antenna is excited by one of the elements in the array (driven element) while other elements are fed through the mutual coupling between them. The phase shifts required for beam steering can be adjusted by changing the reactance of the capacitor loading on the parasitic elements.

Previously, ESPAR investigations have focused on wire [5] and patch elements [7]. Nevertheless, microstrip ESPAR suffers from limited scan range around the boresight. The beam steering angle only achieved the range of $\pm 15^{\circ}$ in [8] and $\pm 20^{\circ}$ in [4]. Besides that, the impedance bandwidth of the microstrip ESPAR is narrow and it is also difficult to maintain the impedance matching during the beam scanning [9]. Recently, dielectric resonator antenna (DRA) ESPAR directly excited by microstrip line has been reported in [10], in which as usual the DRAs were excited in their fundamental mode. There is no much difference on impedance bandwidth between DRA ESPAR in [10] and microstrip ESPAR in [8]. Taking the advantages of higher-order mode DRA to enhance the impedance bandwidth [11] and gain [12] at lower frequencies, the author investigated it at $15 \mathrm{GHz}$.

In this paper, the ESPAR design which consists of a driven DRA and two parasitic DRAs with controlling switches is presented. All DRAs were excited using a higher-order mode. This paper is organized as follows. In Section II, the single element DRA designed using a fundamental mode $\left(T E^{y}{ }_{1 s}\right)$ and a higher-order mode $\left(T E^{y}{ }_{1<3}\right)$, respectively were discussed. In section III, the numerical calculations to get the effective range of capacitance from the DRA ESPAR design were discussed. The ideal switches were then added to the ESPAR DRA design to control the beam scanning. The paper is concluded in Section V.

\section{Single Element Design Of Dielectric Resonator ANTENNA}

The DRA of $T E^{y}{ }_{m \circ n}$ with a dielectric constant, $\varepsilon_{r}=10$ at 15 $\mathrm{GHz}$ can be predicted from the dielectric waveguide model [13]. When the ground plane is present, the modes of the DRA cannot be excited if $n$ is an even number. It is because these modes require the $E$-field to be maximum at $z=0$, which 
is contradictory to the boundary condition that the tangential $E$-field should vanish on the ground plane [14]. Therefore, the index $n$ must be an odd number. The single element DRA has been created, and then simulated using ANSYS High Frequency Structural Simulator (HFSS) software to obtain optimum parameter value. Duroid 5880 dielectric substrate with a thickness of $0.254 \mathrm{~mm}$, a permittivity of 2.2 and a loss tangent of 0.001 was used in the design. Microstrip slot aperture was chosen as the feeding technique because it will give a high directivity and antenna gain to fulfil the $5 \mathrm{G}$ requirements [15].

\section{A. Antenna Configuration}

The configuration of the single element DRA is shown in Fig. 1. Through the simulation and optimization of the design parameters, the optimum parameters are shown in Table I.

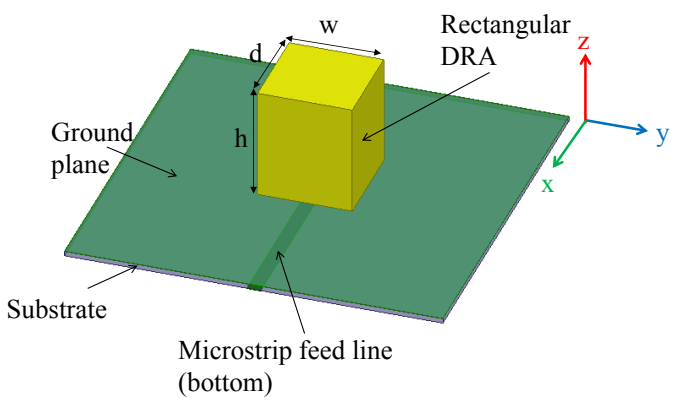

(a)

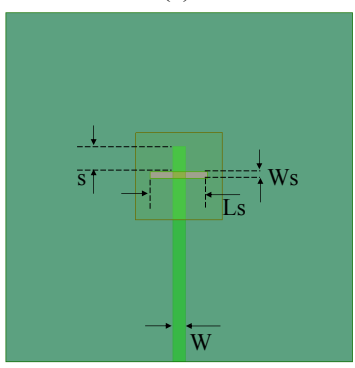

(b)

Fig. 1. Configuration of the single element DRA (a) 3D view (b) Top view

TABLE I. DESIgN PARAMETERS Of ThE Single ElEMENT DRA

\begin{tabular}{|c|c|c|c|c|c|c|c|}
\hline Parameter & $w$ & $d$ & $h$ & $L_{s}$ & $W_{s}$ & $S$ & $W$ \\
\hline $\begin{array}{c}\text { Value (mm) } \\
\text { for } T E^{y}{ }_{1}{ }_{1} 1\end{array}$ & 7.5 & 7.5 & 1.8 & 3.34 & 0.65 & 2 & 0.79 \\
\hline $\begin{array}{c}\text { Value (mm) } \\
\text { for } T E^{y}{ }_{1}{ }^{s 3}\end{array}$ & 4 & 4 & 11.5 & 3.3 & 0.4 & 1.44 & 0.73 \\
\hline
\end{tabular}

\section{B. Comparison of the Simulated Results for Single Element DRA Using Different Excitation Modes.}

1) Magnetic Field Distribution: Fig. 2 depicts the simulated $H_{y}$-field inside the DRA which was excited in $T E^{y}{ }_{1 s l}$ and $T E^{y}{ }_{1 s 3}$ mode. The $H_{y}$ component of the magnetic field is dominant along the center of the DRA. As it is shown in Fig. 2, the different $H$-field distributions inside the DRA were excited using different modes. Obviously, this will affect the antenna bandwidth, radiation pattern and also gain.

2) Reflection Coefficient: The simulated reflection coefficients of the DRA excited in a fundamental mode $\left(T E^{y}{ }_{1 s l}\right)$ and higher-order mode $\left(T E^{y}{ }_{1 s 3}\right)$ are presented in Fig. 3. With reference to the figures, both designs have a good $S_{11}$ at $15 \mathrm{GHz}$. The 10-dB impedance bandwidth has achieved 2.7 $\mathrm{GHz}(13.4 \mathrm{GHz}-16.1 \mathrm{GHz})$ for DRA excited in the $T E^{y}{ }_{1 \cdot 3}$ mode but only $2 \mathrm{GHz}(14.1 \mathrm{GHz}-16.1 \mathrm{GHz})$ was observed for DRA excited in $T E^{y}{ }_{1 s l}$ mode.

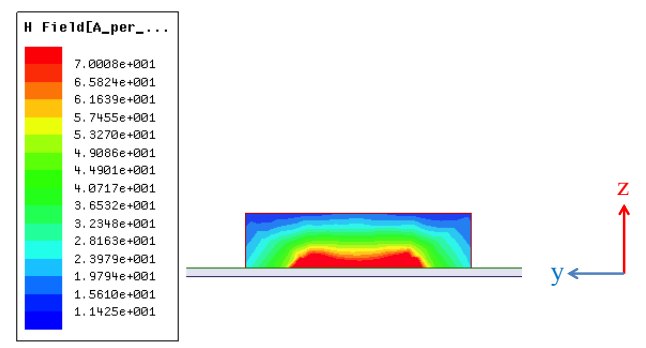

(a)

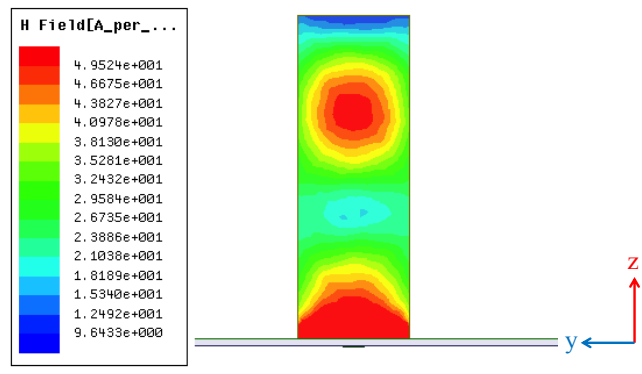

(b)

Fig. 2. Magnetic fields inside the single element DRA (a) $T E^{y}{ }_{1^{\delta} l}$ (b) $T E^{y}{ }_{1^{\delta 3}}$

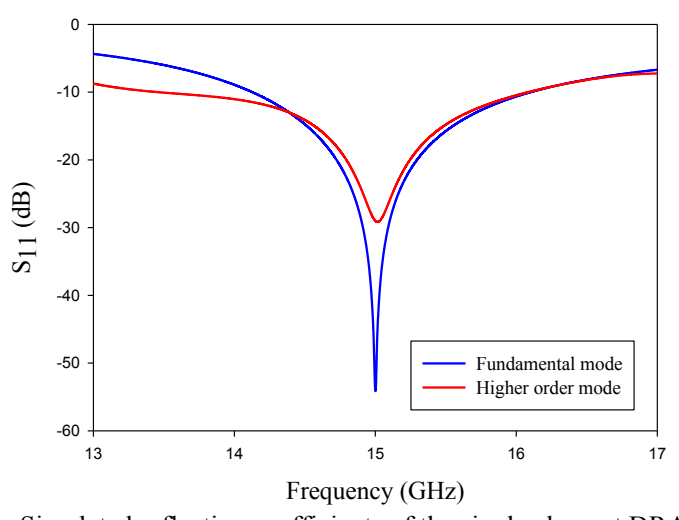

Fig. 3. Simulated reflection coefficients of the single element DRA

3) Radiation Characteristics and Gain: The radiation pattern for $E$-plane and $H$-plane are compared in Fig. 4. As it can be seen, both designs have a broadside radiation. Nevertheless, the DRA excited in $T E^{y}{ }_{103}$ mode has more than one equivalent magnetic dipole along the height [14]. The separation between the equivalent magnetic dipoles is determined by the DRA dimensions. Therefore, a narrower 
beamwidth can be achieved with a DRA excited in $T E^{y}{ }_{183}$ mode, in comparison with the DRA excited in $T E^{y}{ }_{1 s l}$ mode. Besides that, the gain for the single DRA excited in $T E^{y}{ }_{1 \cdot 3}$ mode has achieved $9.9 \mathrm{dBi}$ in comparison to $5.6 \mathrm{dBi}$ when it was excited in $T E^{y}{ }_{1 s l}$ mode.

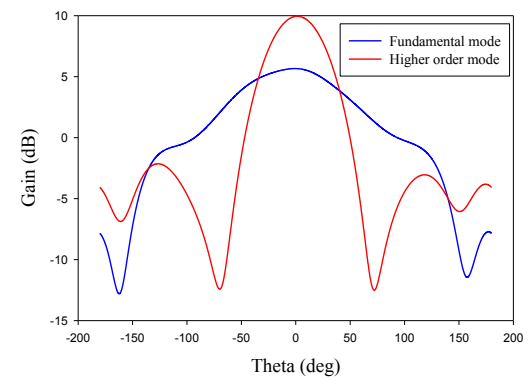

(a)

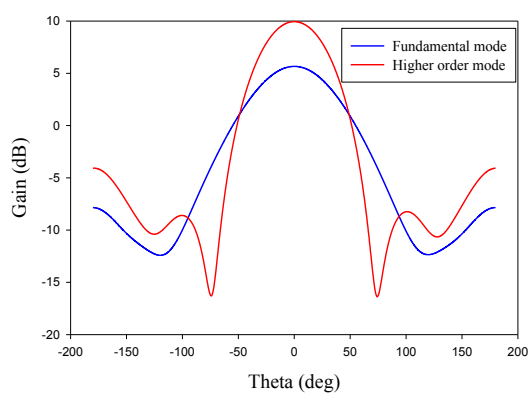

(b)

Fig. 4. Simulated radiation patterns of the single element DRA (a) E-plane (xz plane) (b) H-plane (yz plane)

\section{DRA WITH PARASITIC ELEMENTS AND CAPACITOR LOADING}

The comparison of simulated results in Section II shows the advantages of using a higher-order mode $\left(T E^{y}{ }_{133}\right)$ in $5 \mathrm{G}$ applications. DRA with parasitic elements and capacitor loading was then examined by using $T E_{103}^{y}$ mode. Besides that, numerical calculations were also developed in this section to study the capacitance range that will affect the steering angle.

\section{A. Theory and Design}

The design in Fig. 5 consists of three elements of dielectric resonator. The driven DR at port 1 was fed by an RF source and the parasitic DR at port 2 and 3 were excited by the driven DR through the mutual coupling. The mutual coupling was determined by the distance between the dielectric resonator elements and the phase shifts between the elements, which have been adjusted by changing the reactance of $C_{A}$ and $C_{B}$. The best distance, $d$ between the elements is $0.4 \lambda$.

This array can be analyzed with three-port network theory in [4], with the help of $Z$ matrix in (1):

$$
\left(\begin{array}{l}
V_{1} \\
V_{2} \\
V_{3}
\end{array}\right)=\left(\begin{array}{lll}
Z_{11} & Z_{12} & Z_{13} \\
Z_{21} & Z_{22} & Z_{23} \\
Z_{31} & Z_{32} & Z_{33}
\end{array}\right)\left(\begin{array}{l}
I_{1} \\
I_{2} \\
I_{3}
\end{array}\right)
$$

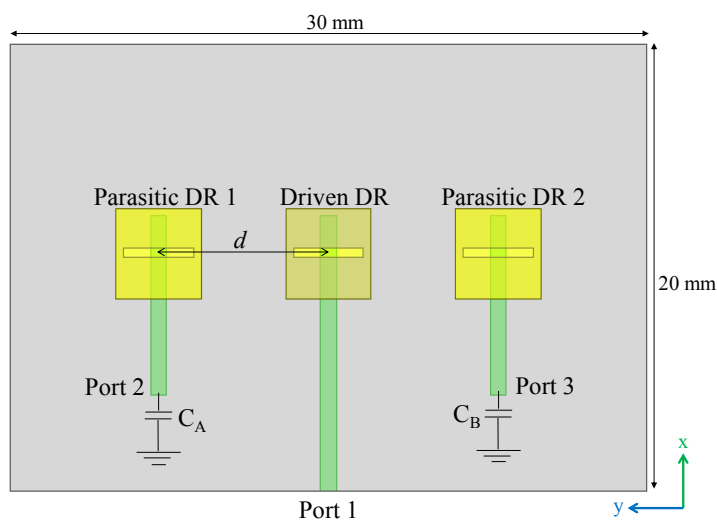

Fig. 5. Configuration of DRA with parasitic elements and capacitor loading

By using (1), the ratio between the current on the two parasitic DRs and the current on the driven DR was obtained, as shown in (2).

$$
\left(\begin{array}{l}
\frac{I_{2}}{I_{1}} \\
\frac{I_{3}}{I_{1}}
\end{array}\right)=\left(\begin{array}{cc}
Z_{22}+Z_{C A} & Z_{23} \\
Z_{32} & Z_{33}+Z_{C B}
\end{array}\right)^{-1}\left(\begin{array}{l}
-Z_{21} \\
-Z_{31}
\end{array}\right)
$$

The radiation pattern of the phased array was calculated by multiplying the array factor by the radiation pattern of a single DRA. The array factor of the three DRA was found to be:

$$
A F=1+\frac{I_{2}}{I_{1}} e^{-j k d \cos \theta}+\frac{I_{3}}{I_{1}} e^{j k d \cos \theta}
$$

where $k$ is the wavenumber and $d$ is the center to center distance between the DRAs.

\section{B. Effective Capacitance Range}

As stated, the capacitances $C_{A}$ and $C_{B}$ are the key controls for the radiation pattern. By adjusting their values, the input impedance of the parasitic DRs can be controlled and this in turn will allow the controlling of the current distributions on them. First, the predicted value for effective range of capacitances $C_{A}$ and $C_{B}$ for the design in Fig. 5 was obtained by determining the reflection coefficient, $\Gamma$ in (4).

$$
\Gamma=\frac{Z_{\text {in }}-Z_{C}}{Z_{\text {in }}+Z_{C}}
$$

where $Z_{C}=1 / j \omega C$ is the reactance provided by either capacitor $C_{A}$ or $C_{B}$ and the input impedance, $Z_{\text {in }}$ can be calculated as in (5):

$$
Z_{\text {in }}=Z_{11}+Z_{12} \frac{I_{2}}{I_{1}}+Z_{13} \frac{I_{3}}{I_{1}}
$$

The values for all $Z$ parameters involved in these numerical calculations were obtained from ANSYS HFSS. Then, by 
using MATLAB, the reflection coefficient, $\Gamma$ was calculated for parasitic DR at port 2 by varying capacitance $C_{A}$. Fig. 6 shows the magnitude and phase of $\Gamma$ versus $C_{A}$ in which no change was noted in $|\Gamma|$ and $\Gamma_{\phi}$ when the capacitance exceeds 1 $\mathrm{pF}$. The same thing happens when $C_{B}$ is varied. Since $|\Gamma|$ is high when $C_{A}<0.01 \mathrm{pF}$ and nearly flat when $C_{A}>1 \mathrm{pF}$, the range of $0.01 \mathrm{pF}-1 \mathrm{pF}$ was considered as the effective capacitances that would affect the radiation pattern of the proposed antenna.

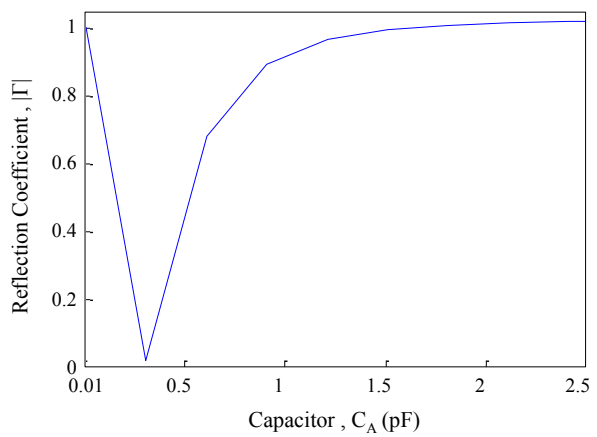

(a)

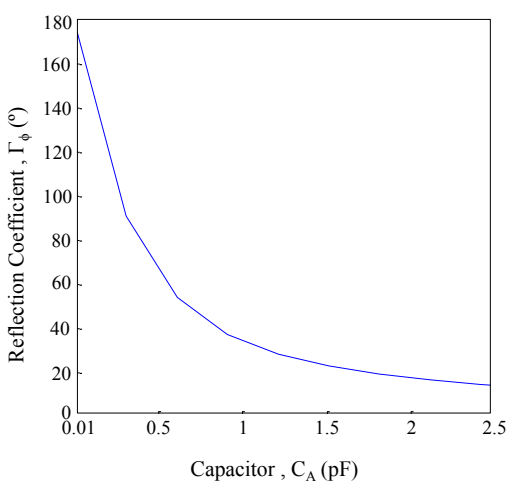

(b)

Fig. 6. Variation of $\Gamma$ with capacitance $\mathrm{C}_{\mathrm{A}}$ (a) magnitude of $\Gamma$ (b) phase of $\Gamma$

\section{SWITCHED PARASITIC DRA With CAPACITOR LOADING}

\section{A. Proposed Design}

A directional beam was formed by an appropriate choice of the parasitic element termination, and the beam was steered by switching the termination on the parasitic elements. Fig. 7 shows the top view layout of the proposed switched parasitic DRA to perform five different steering angles. The switching configuration for antenna beam steering is shown in Table II. The ON and OFF states refer to the short-circuit and opencircuit between the corresponding capacitor and the parasitic element, respectively. The shorting pin was used to connect the ground plane.

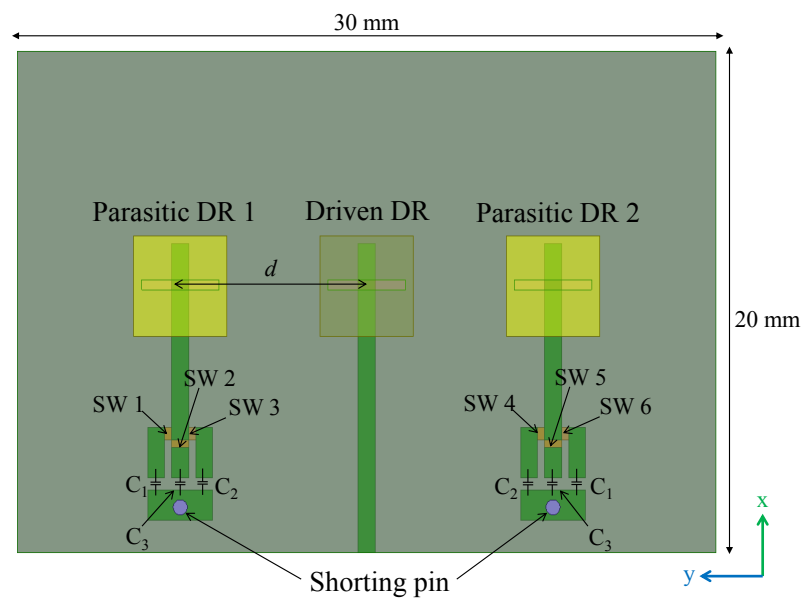

Fig. 7. Configuration of switched parasitic DRA with capacitor loading

TABLE II. SWITCH CONFIGURATION For ANTENNA BEAM STEERING

\begin{tabular}{|c|c|c|c|c|c|c|}
\hline & \multicolumn{3}{|c|}{ Parasitic DR1 } & \multicolumn{3}{|c|}{ Parasitic DR2 } \\
\hline & $\begin{array}{c}C_{I}=0.01 \\
p F\end{array}$ & $\begin{array}{c}C_{3}=1 \\
p F\end{array}$ & $\begin{array}{c}C_{2}=0.1 \\
p F\end{array}$ & $\begin{array}{c}C_{2}=0.1 \\
p F\end{array}$ & $\begin{array}{c}C_{3}=1 \\
p F\end{array}$ & $\begin{array}{c}C_{l}=0.01 \\
p F\end{array}$ \\
\hline & $S W 1$ & $S W 2$ & SW3 & SW4 & SW5 & SW6 \\
\hline Case I & OFF & $\mathrm{ON}$ & OFF & OFF & OFF & $\mathrm{ON}$ \\
\hline Case II & OFF & OFF & ON & OFF & OFF & $\mathrm{ON}$ \\
\hline Case III & $\mathrm{ON}$ & OFF & OFF & $\mathrm{OFF}$ & OFF & $\mathrm{ON}$ \\
\hline Case IV & $\mathrm{ON}$ & OFF & OFF & ON & OFF & OFF \\
\hline Case V & $\mathrm{ON}$ & OFF & OFF & OFF & $\mathrm{ON}$ & OFF \\
\hline
\end{tabular}

\section{B. Simulated Result}

1) Reflection Coefficient, $S_{11}(d B)$ : The simulated reflection coefficient is shown in Fig. 8. These results indicate an insignificant variation of impedance matching in different cases which does not affect the normal operation of the antenna as expected. The $-10 \mathrm{~dB}$ impedance bandwidth has achieved $2.6 \mathrm{GHz}$ in the range of $13.7 \mathrm{GHz}-16.3 \mathrm{GHz}$ for all cases.

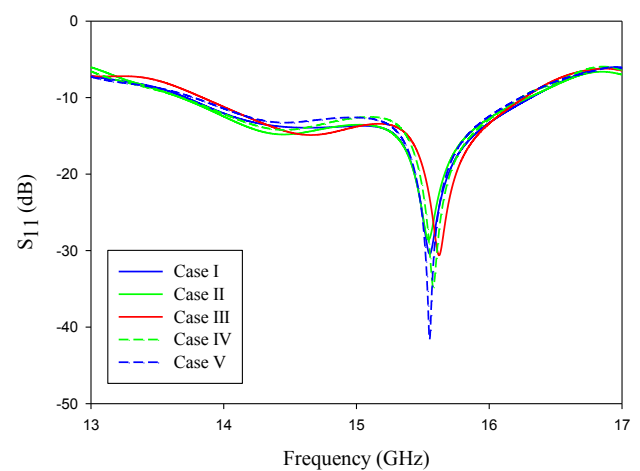

Fig. 8. Simulated reflection coefficients for different cases as mentioned in Table II 
2) H-plane Radiation Pattern and Gain: The beam steering angle (relative to the boresight) for five different cases is illustrated in Fig. 9. It was observed that when $S W 2$ was terminated with $C_{3}=1 \mathrm{pF}$ and $S W 6$ was terminated with $C_{I}=0.01 \mathrm{pF}$, the beam has reached its maximum coverage at $26^{\circ}$. When the capacitance values were alternated, in which $S W 1$ was terminated with $C_{1}=0.01 \mathrm{pF}$ while $S W 5$ with $C_{3}=1$ $\mathrm{pF}$, the steering angle was found to shift back to $26^{\circ}$. The maximum coverage of beam was recorded at $0^{\circ}$ when both $S W 1$ and $S W 6$ were terminated with $C_{l}=0.01 \mathrm{pF}$. The simulated peak gain and scan angle for different cases are shown in Table III. The gain was found to decrease when the scan angle was increased due to the emergence of side lobe.

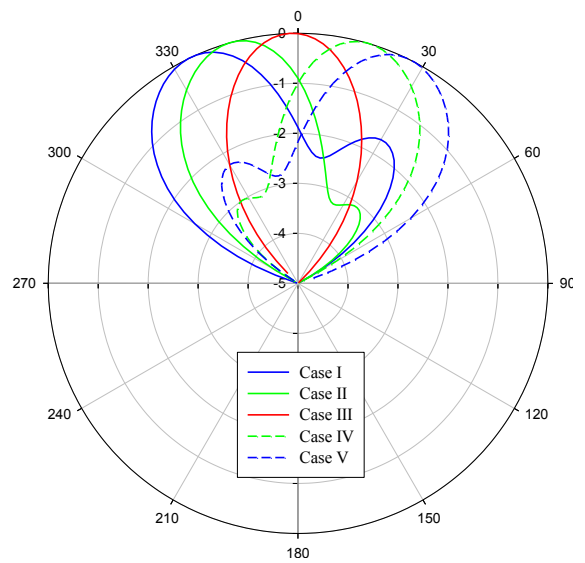

Fig. 9. Simulated radiation patterns for different cases as mentioned in Table II.

TABLE III. SimUlated PEAK GAIN AND SCAN ANGLE For DIFFERENT CASES

\begin{tabular}{|c|c|c|c|c|c|}
\hline & Case I & Case II & Case III & Case IV & Case V \\
\hline Peak gain (dBi) & 7.8 & 8.3 & 8.9 & 8.4 & 8.0 \\
\hline Scan angle (deg) & -26 & -16 & 0 & +16 & +26 \\
\hline
\end{tabular}

\section{CONCLUSION}

A new switched parasitic DRA excited in a higher-order mode has been demonstrated using switching technique at capacitor loading. It should be noted that impedance matching was maintained at all beam steering angles and a gain of more than $7.7 \mathrm{dBi}$ has been achieved. Using a higher-order mode DRA allows for a wider bandwidth and a higher gain. With this design, it is possible to achieve $\pm 26^{\circ}$ scanning capabilities without using phase shifter. It is believed that this new phased array approach is the enabling technique for $5 \mathrm{G}$ low cost phased arrays. Further exploration into how a higher gain and a larger steering angle can be achieved is being carried out by the authors.

\section{ACKNOWLEDGMENT}

The authors would like to thank the Ministry of Education (MOE) for sponsoring the PhD study at Universiti Teknologi Malaysia (UTM). Also, special thanks go to MOE and UTM for sponsoring this work under FRGS (Vot 4F733 and 4F283), UTM GUP (Vot 05H62\&11H59) and MG (00M69).

\section{REFERENCES}

[1] F. Boccardi, R. W. Heath, A. Lozano, T. L. Marzetta and P. Popovski, "Five Disruptive Technology Directions for 5G," IEEE Commmunication Magazine, 2014

[2] J. Rodriguez, Fundamentals of 5G Mobile Network. United Kingdom: Wiley, 2015, pp.242

[3] "Spectrum above $6 \mathrm{GHz}$ for future mobile communications".[Online].Available: http://stakeholders.ofcom.org.uk/ binaries/consultations/above6ghz/summary/spectrum_above_6_GHz_CFI.pdf.

[4] Y. Yusuf and X. Gong, "A low-cost patch antenna phased array with analog beam steering using mutual coupling and reactive loading," IEEE Antennas Wireless Propag, Lett., vol. 7, pp. 81-84, 2008.

[5] H. Kawakami and T. Ohira, "Electronically steerable passive array radiator (ESPAR) antennas," IEEE Antennas Propag. Mag., vol. 47, pp. 43-50, 2005.

[6] M. R. Islam and M. Ali, "Elevation plane beam scanning of a novel parasitic array radiator antenna for $1900 \mathrm{MHz}$ mobile handheld terminals," IEEE Trans. Antennas Propag., vol. 58, pp. 3344-3352, 2010.

[7] D. T. Nguyen, R. Siragusa and S. Tedjini, "Beam steering patch antenna using reactive loading and yagi-antenna concept," Microwave and Optical Technology Letters., vol. 57, pp. 417-421, 2015.

[8] J. J. Luther, S. Ebadi and X. Gong, "A microstrip patch electronically steerable parasitic array radiator (ESPAR) antenna with reactance-tuned coupling and maintained resonance," IEEE Trans. Antennas Propag., vol. 60 , pp. $1803-1813,2012$.

[9] M. R. Islam and M. Ali, "A $900 \mathrm{MHz}$ beam steering parasitic antenna array for wearable wireless application," IEEE Trans. Antennas Propag., vol. 61, pp. 4520-4527, 2013.

[10] M. R. Nikkah, J. Rashed-Mohassel, and A. A. Kishk, "Compact lowcost phased array of dielectric resonator antenna using parasitic elements and capacitor loading," IEEE Trans. Antennas Propag., vol. 61, pp. 2318-2321, 2013.

[11] G. B. Babik, C. D. Nallo, and A. Faraone, " Multimode dielectric resonator antenna of very high permittivity," IEEE Antennas Propagation Symposium, vol. 2, pp. 1383-1386, 2004.

[12] A. Petosa, S. Thirakoune, and A. Ittipiboon, "Higher-order modes in rectangular DRAs for gain enhancement," $13^{\text {th }}$ International Symposium on Antenna Technology and Applied Electromagnetics, 2009.

[13] R. K. Mongia, and A. Ittipiboon, "Theoretical and experimental investigation on rectangular dielectric resonator antenna," IEEE Trans. Antennas Propag., vol. 45, pp. 1348-1356, 1997.

[14] K. M. Luk and K. W. Leung, Dielectric Resonator Antenna, England: Research Studies Press, 2002.

[15] N. H. Shahadan, M. R. Kamarudin, N. A. Zainal, J. Nasir, M. Khalily, and M. H. Jamaluddin, "Investigation on Feeding Techniques for Rectangular Dielectric Resonator Antenna in Higher-Order Mode for 5G Applications,"'Applied Mechanics and Materials, vol. 781, pp. 4144, August 2015. 